\title{
Formación de capacidades para fomentar el diálogo entre culturas, a través de las TIC en Medellín (Colombia)*
}

\author{
Gabriel Jaime Lotero Echeverri**
}

\section{Resumen}

Introducción. Estudio sobre la aplicación de una propuesta formativa, en modalidad b-learning (aprendizaje bimodal), sobre el diseño de contenidos para la web en el tema de la diversidad cultural. Objetivo. Su propósito es analizar su pertinencia en la creación y fortalecimiento de capacidades para una comunicación digital que fomente el diálogo intercultural, en la comunidad universitaria. Materiales y métodos. El contexto en el cual surge la propuesta es la ciudad de Medellín, que acoge migraciones provenientes de la región y el país, debido a diversos motivos, entre los cuales se incluye el conflicto interno colombiano. Como referentes teóricos se destacan los estudios sobre la diversidad cultural, así como el reconocimiento de condiciones estructurales de la región. Se exponen los momentos metodológicos, a partir de estrategias propias del enfoque bimodal, el cual integra didácticas tanto presenciales como propias de escenarios digitales. Resultados. Los resultados de la aplicación del diseño contribuyen a conocer mejor la diversidad en la comunidad universitaria, así como la postura de los estudiantes ante la misma. Conclusión. Entre las conclusiones se destaca la importancia del reconocimiento y la necesidad de ir más allá de un abordaje instrumental de la comunicación digital, para profundizar en la comprensión de temáticas complejas como la diversidad cultural.

Palabras clave: comunicación, tecnologías de la información, diversidad cultural, educación a distancia.

\author{
Formation of competencies for boosting \\ intercultural dialogue by means of IT in \\ Medellín, Colombia
}

\begin{abstract}
Introduction. This is a study about a formative proposal with a b-learning model (bimodal learning) on the design of contents for the web in the cultural diversity subject. Objective. To analyze its relevance in the creation and the strengthening of competencies for a digital communication that boosts intercultural dialogue in the university community. Materials and methods. The context in which the proposal emerges is Medellín, a city that receives migrations from the region and the country due to several reasons, among which the Colombian internal conflict is included. As theoretical references cultural diversity studies can be remarked, and also the knowledge on the structural conditions of the region. The methodological moments are exposed from those strategies proper of the bimodal focus, which integrates online and offline didactic scenarios. Results. The results of the application of the design contribute to achieve better knowledge of the diversity within the university community and the students' positions concerning it. Conclusion. Among the conclusions, the importance of the recognition and the necessity of an approach beyond that of the instrumental one about digital communication are remarked in order to delve into the comprehension of complex subjects, such as cultural diversity.
\end{abstract}

Key words: communication, IT, cultural diversity, online education

\footnotetext{
* Artículo original resultado del proyecto de investigación "Estudio de tendencias del periodismo digital en la sociedad red" financiada por la fundación Universitaria Luis Amigó de Medellín.

** Comunicador Social (Universidad de Antioquia), máster en Mediación Intercultural (Universidad de Valencia, España) y máster en Comunicación y Educación de la UNED. Docente de la Fundación Universitaria Luis Amigó. Miembro del Grupo de Investigación Urbanitas (Medellín, Colombia). Correo electrónico: gabriel.loteroec@amigo.edu.co
} 


\section{Formação de capacidades para fomentar o diálogo entre culturas, através das TIC em Medellín (Colômbia)}

\section{RESUMO}

Introdução. Estudo sobre a aplicação de uma proposta formativa, em modalidade b-learning (aprendizagem bimodal), sobre o desenho de conteúdos para a web no assunto da diversidade cultural. Objetivo. Seu propósito é analisar sua pertinência na criação e fortalecimento de capacidades para uma comunicação digital que fomente o diálogo intercultural, na comunidade universitária. Materiais e métodos. O contexto no qual surge a proposta é a cidade de Medellín, que acolhe migrações provenientes da região e do país, devido a diversos motivos, entre os quais se inclui o conflito interno colombiano. Como referentes teóricos se destacam os estudos sobre a diversidade cultural, assim como o reconhecimento de condições estruturais da região. Se expõe os momentos metodológicos, a partir de estratégias próprias do enfoque bimodal, o qual integra didáticas tanto presenciais como própria de cenários digitais. Resultados. Os resultados da aplicação do desenho contribuem a conhecer melhor a diversidade na comunidade universitária, assim como a postura dos estudantes ante a mesma. Conclusão. Entre as conclusões se destaca a importância do reconhecimento e a necessidade de ir mais além de uma abordagem instrumental da comunicação digital, para aprofundar na compreensão de temáticas complexas como a diversidade cultural.

Palavras chave: comunicação, tecnologias da informação, diversidade cultural, educação a distância.

\section{Introducción}

Se presentan los resultados de la aplicación de una propuesta formativa, en modalidad b-learning, en el campo de la promoción del diálogo intercultural. Esta propuesta formativa es concebida a partir del reconocimiento de la situación de diversidad étnica, así como de la necesidad de fortalecer las competencias de profesionales de la comunicación, en formación, para fomentar el diálogo intercultural, tal y como lo requiere la sociedad; por ello se enmarca en el campo de la etnoeducación, enfoque desde el cual se reconoce el derecho de las comunidades étnicas a que su cultura sea incluida en los currículos de las instituciones educativas a las cuales asisten (Carabalí, 2007). Pero, en esta propuesta se va más allá en cuanto se concibe la etnoeducación como una necesidad de la sociedad en su conjunto, de manera que todos los estudiantes deben ser sensibilizados.

El programa se enfoca en las necesidades de la población afrodescendiente como una comunidad en situación de vulnerabilidad. Se propone fortalecer las competencias investigativas y comunicativas, con el fin de visibilizar procesos comunitarios, sensibilizar a la comunidad en general, denunciar e incidir en la construcción de políticas para la adecuada gestión de la diversidad. Se trata de una propuesta enmarcada en la formación ciudadana, el fomento del pluralismo, dirigida a estudiantes de comunicación, para contribuir con la comprensión y la visibilidad de esta temática.

\section{Fundamentación teórica}

Colombia es un Estado social de derecho (Constitución Política de 1991). Sin embargo, no ha sido posible construir en la realidad de toda la ciudadanía lo que se recoge en el papel. Esta imposibilidad se explica, de una parte, por el nudo de las múltiples formas de violencia que sufre el país desde hace décadas, pero también está imbricada en la estructura social profundamente injusta que lleva a Colombia a encabezar los índices internacionales de los territorios con mayor desigualdad del planeta (PNUD, 2010).

Uno de los efectos perversos de la pobreza, en sus múltiples dimensiones, es la amenaza que representa para el disfrute pleno de la ciudadanía, para incidir en la toma de decisiones que afectan la vida personal y colectiva (Sen, 2007). En este sentido, vale la pena reconocer que el desarrollo humano parte de la centralidad de la persona como 
protagonista de su propio desarrollo (Sen, 2000).

Pero, merece la pena preguntarse en qué medida las aspiraciones, sueños y proyectos de comunidades y personas son el resultado de procesos de larga duración. Esto incide en las expectativas con las cuales llegan los estudiantes pertenecientes a una minoría étnica, en sus auto-percepciones identitarias. La auto-percepción no es suficiente como criterio para conocer la composición de una población ni la situación de inclusión social, ya que las personas y las comunidades realizan un proceso de adaptación a sus circunstancias, por medio del cual se percibe como natural una situación de injusticia (Pereira, 2009, 57). Por ejemplo, en Colombia se ha posicionado una imagen del afrodescendiente como una persona alegre, de fiestas y con gran vocación para dedicarse a la música o, por otro lado, como un buen deportista. Y no es extraño que sean estas dos las vías de ascenso social que se les facilitan.

La situación de vulnerabilidad ante la exclusión social es multidimensional y compleja. En la sociedad contemporánea otro factor de riesgo importante en este sentido es el acceso y el aprovechamiento de las oportunidades que representan las TIC, que no están al alcance de todos en Colombia. Esta nueva brecha, conocida como brecha digital, viene a reforzar la histórica y persistente exclusión social y la vulnerabilidad de personas y colectivos enteros (ECLAC, 2013).

\section{Estudios de la diversidad cultural}

Podemos entender cultura como modos de vida, como información heredada por aprendizaje social, por imitación, comunicación y enseñanza (Ariño, 1997, 41). Se destacan las siguientes características de cultura: es constitutiva de todo ser humano, es compartida, produce homogeneidad y comunidad, existen tantas culturas como grupos humanos (Ariño, 1997, 45).
Existe diversidad tanto intercultural, como intracultural, determinada por factores como la historia, el género, la edad, la ocupación, la clase social, la educación (Ariño, 1997, 62). Es pertinente relacionar la diversidad cultural con la desigualdad social, desigualdad que genera relaciones de dominación, dependencia y concentración de recursos y oportunidades, como una relación asimétrica entre diferentes, sean estos los privilegiados o los excluidos (García-Canclini, 1982). Al abordar un tema como la diversidad étnica es necesario considerar que la relación entre la desigualdad y la diversidad se actualiza en nuevos desequilibrios, a manera de acumulación de desventajas: "son desempleados, pobres, migrantes indocumentados, homeless, desconectados. Para muchos millones el problema no es mantener 'campos sociales alternos', sino ser incluidos" (García-Canclini, 2004, 119).

En un mundo globalizado, es aún más difícil establecer culturas diferenciadas, territorializadas en Estados-nación (Hernández, 2005). Cuando las dinámicas del sistema mundial expulsan y atraen a millones de personas a otros lugares, en busca de oportunidades (desde hace siglos y en flujos dinámicos), cuando en las sociedades de acogida confluyen personas con otras visiones del mundo, cobra vigencia la interculturalidad, como superación del etnocentrismo de quienes miran lo diferente como atraso (García-Canclini, 2004, 126). Interculturalidad como modelo de integración, como un tipo de relación basado en el mutuo respeto, inspirado en valores como la dignidad humana, del cual derivan los derechos humanos ${ }^{1}$.

\section{Educación y ciudadanía en un contexto global}

Pensar la educación exige reconocer que la globalización ${ }^{2}$ no solo tiene efectos económicos en los territorios en los cuales se interviene; es necesario indagar por sus efectos en la cultura, en la construcción de identidad y

\footnotetext{
1 Desde una concepción de integralidad, que incluye los derechos civiles y políticos junto con los derechos económicos, sociales y culturales. Ver: ONU. (1966a), (1966b).

2 Este componente se desarrolla en el artículo: Lotero, G. (2014b).
} 
en el fortalecimiento de valores universales como la tolerancia. Fomentar la formación en competencias ciudadanas potencia las capacidades de la persona para disfrutar de sus derechos fundamentales y para saber exigir su cumplimiento (Ministerio de Educación de Colombia, 2004, 6).

Debido a la transversalidad de estos procesos de formación, el periodismo es un actor importante en la construcción de ciudadanía, y en su condición de veedor también asume la investigación y denuncia de las situaciones que niegan la equidad y la justicia. El ejercicio del periodismo se ha visto transformado ante la popularización de las TIC, y los procesos de convergencia de los diversos soportes de la información (con todas las posibilidades expresivas de la multimedialidad que integra textos, vídeos, imágenes, entre otros recursos), potenciados por los escenarios digitales, los cuales han transformado los modelos comunicativos, de manera que los usuarios están habilitados también como productores de contenido (interactividad) que en la virtualidad se configuran bajo una arquitectura de tipo hipertextual, que rompe con la linealidad de los mensajes $^{3}$.

Ante la profundidad de las transformaciones y desafíos que los escenarios digitales representan a quienes se están formando como profesionales del periodismo, se plantea la necesidad de acudir a concepciones pedagógicas que sean consistentes con la naturaleza activa requerida tanto al profesional como a sus públicos. En este sentido, el aprendizaje bimodal (blended learning) es una estrategia de incorporación de las tecnologías de la información y la comunicación (TIC) a procesos educativos, que se basa en la integración de "múltiples formas de enseñanza, por medio del uso de recursos físicos y virtuales" (Araya, 2007, 3). Se ha planteado como alternativa al e-learning, ya que permite atender a algunas de las limitaciones que este representa, especialmente cuando se pretende involucrar a públicos con un nivel bajo de competencias mediáticas o cuando estos participantes puedan sentirse desmotivados y aislados ante las exigencias propias de los espacios de formación meramente virtuales (Cabero y Llorente, 2008).

\section{Contexto del proyecto}

Medellín es la segunda ciudad de Colombia, con cerca de 3.500 .000 habitantes (Área Metropolitana), según el Departamento Administrativo Nacional de Estadística (DANE). Y es una de las ciudades más desiguales del país, con un índice de pobreza de $22 \%$, medido por satisfacción de necesidades básicas (Red de Ciudades Cómo Vamos, 2014). En cuanto a la composición etnográfica, según datos del último censo nacional del DANE, el $79 \%$ de la población no se reconoce como perteneciente a una etnia determinada; el $19 \%$, como afrocolombiano, y el $2 \%$, como indígena (DANE, 2007).

La ciudad acoge migraciones internas. Se estima que cerca del $40 \%$ de la población de Medellín nació en otro lugar del país. Muchos son desplazados por el conflicto armado interno que padece Colombia desde hace más de 50 años (Alcaldía de Medellín, 2010). En Colombia el acceso a la Educación Superior no está al alcance de todos ${ }^{4}$. Además del acceso, existe un problema de deserción importante, cercana al $12 \%$, según datos del Ministerio de Educación Nacional (Guzmán, C., 2009).

La institución de Educación Superior en la que se desarrolla el proyecto es de carácter privado y católico, y cuenta con cerca de 10 mil estudiantes. Por su esquema de precios, sus programas están al alcance de gran diversidad de públicos, incluyendo estudiantes de sectores desfavorecidos que acceden a becas sociales. Se estima que el $10 \%$ de los colombianos son afrodescendientes (DANE, 2007), muchos estudiantes afro de la institución provienen de distintas regiones (algunos de ellos de regiones

3 Este componente se desarrolla en el capítulo de libro: LOTERO, G. (2014a).

4 En Colombia, en el año 2012, el 40 \% de la población entre 17 - 21 años de edad están matriculadas en programas de formación superior, según datos del Sistema Nacional de Información de la Educación Superior del Ministerio de Educación Nacional, disponibles en www.mineducacion.gov.co 
rurales apartadas, que tienen menos acceso a oportunidades y recursos).

\section{Materiales y métodos}

La metodología se basa en el enfoque constructivista, a partir de la identificación de saberes previos, expectativas e intereses del grupo de estudiantes, su consumo de medios, a través de cuestionarios y de un taller de producción de contenidos para la web.

Se diseña una unidad didáctica, desarrollada a partir de una serie de ejercicios prácticos individuales y grupales, a través de los cuales los estudiantes van incorporando los principios básicos de la producción de contenidos para Internet: hipertextualidad, multimedialidad, interactividad y usabilidad.

Los talleres y ejercicios del programa de formación no solo se basan en la clase magistral sino que se apoyan en guías, rúbricas y bibliografía, disponibles en diferentes escenarios virtuales, en los cuales los estudiantes deben evidenciar su propia producción, aprovechando como herramientas la plataforma Moodle (especialmente los foros para las asesorías), el sitio web del docente, el sitio web de cada estudiante, las bases de datos de Ebsco ${ }^{\circledR}$ y los recursos complementarios que los estudiantes busquen en Internet.

La temática central trabajada en la unidad didáctica es el enfoque del diálogo intercultural, del cual los estudiantes investigan referentes y antecedentes importantes, en bases de datos especializadas, disponibles en la plataforma Ebsco $\circledast$, como un insumo documental para producir contenidos para su sitio web.

Los estudiantes también deben responder una encuesta, a partir de un instrumento propuesto y explicado por el docente, el se sistematiza en GoogleDocs $₫$, para su posterior interpretación. Se acude a un muestreo intencionado, según el cual se debe consultar a personas que estudien actualmente en la institución universitaria y que sean afrodescendientes.

Los estudiantes exponen sus reportajes electrónicos y los sustentan. Se dinamiza un debate. $Y$ posteriormente responden un cuestionario de autoevaluación en línea.

\section{Resultados y discusión}

En el segundo semestre de 2014, se implementa la presente propuesta formativa, durante el inicio de la asignatura "Periodismo Virtual". En la primera sesión, cada estudiante responde dos cuestionarios de identificación de saberes previos, relacionados con su aproximación a las TIC y a la redacción periodística, y su consumo de medios e Internet. Se destacan los siguientes resultados:

Los grupos de estudiantes del curso están conformados por un $77 \%$ de mujeres y un $23 \%$ de hombres. Se destaca que el $42 \%$ tiene entre 17 y 20 años y el $39 \%$ tiene entre 21 y 25 años. El $52 \%$ está cursando el quinto semestre del pregrado y el $36 \%$, está en un nivel superior, por lo que ya han superado buena parte de los cursos de escritura y redacción periodística. Al preguntarles por el tiempo destinado a navegar en Internet, más de la mitad accede más de 5 horas semanales a Internet.

\section{Resultados del Taller de Comunicación Intercultural}

Primero, cada estudiante responde unas "preguntas iniciales", relacionadas con la temática, disponibles en un formulario en el sitio web del docente. Se destacan los siguientes resultados:

Al preguntarles si existen las razas, el $83 \%$ de los estudiantes afirma que sí, mientras el 17 $\%$ responde que no. Luego se indaga por la manera en la cual cada estudiante se identifica, sin dar opciones de respuesta predeterminada; los estudiantes responden como se aprecia en la figura 1:

Se destaca que un $17 \%$ se identifican como pertenecientes a la raza humana, lo que permite inferir que se va avanzando en el discurso de la igualdad.

En un segundo momento, se les pregunta si se consideran racistas, a lo que el $98 \%$ responde que no. Sin embargo, cuando se les pregunta 
si existe el racismo en Colombia, el $97 \%$ responde que sí; e incluso, el $17 \%$ responde que se ha sentido discriminado en alguna ocasión por esta razón.

\section{¿A cuál raza pertenece usted?}

\section{Afrodescendiente}

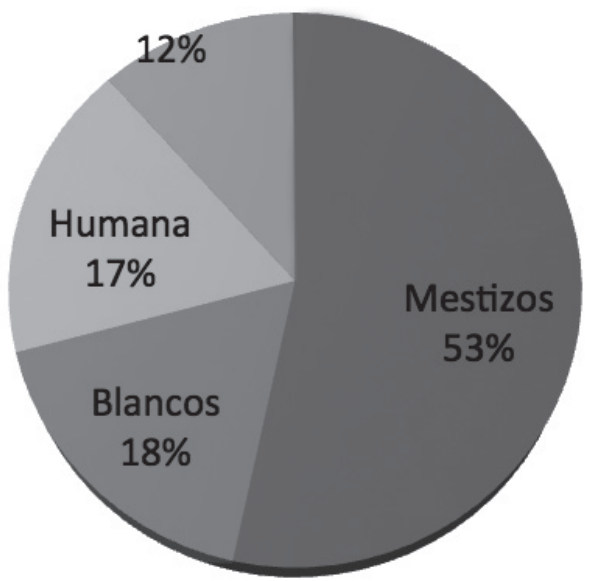

Figura 1. Auto-identificación de los estudiantes participantes

\section{Desarrollo del taller}

El taller inicia con la proyección intencionada del vídeo de sensibilización "Lekk" del cual se genera un debate con el grupo y los estudiantes reconocen con facilidad el cuestionamiento a la discriminación basada en los prejuicios y estereotipos de raza o etnia. A continuación, se les presenta un vídeo informativo sobre el racismo. Después, se revisa el caso de la medida de discriminación positiva (acción afirmativa) por la cual la comunidad afrocolombiana cuenta con dos congresistas. Este caso se revisa a partir de dos medios electrónicos periodísticos colombianos, que cubren la elección de representantes de las comunidades afrodescendientes, que en las últimas elecciones no pudieron posesionarse porque la elección fue demandada debido a que los dos elegidos no son negros ni han trabajado por los derechos de la comunidad afro. Se destaca que por corrupción, por ignorancia o por desinterés por la política, estas dos curules terminan en poder de personas que no representan a la comunidad afro, según informa la prensa.

A continuación, se les explica a los estudiantes la investigación que deben realizar, tanto con fuentes directas como secundarias, y la producción en la cual deben presentar los resultados de la misma, pensados para ser publicados en un sitio web, aplicando los criterios de hipertextualidad, multimedialidad e interactividad trabajados anteriormente.

\section{Resultados de la encuesta a estudiantes afrodescendientes}

En pareja, los estudiantes aplicaron el cuestionario (anteriormente explicado por el docente). Fueron encuestados 55 estudiantes, de los cuales el $56 \%$ son mujeres. Como se observa en la figura 2, proceden en su mayoría de regiones del país que históricamente tienen presencia significativa de población afrodescendiente:

$5 \quad$ Disponible en Youtube ${ }^{\circledR}$ 


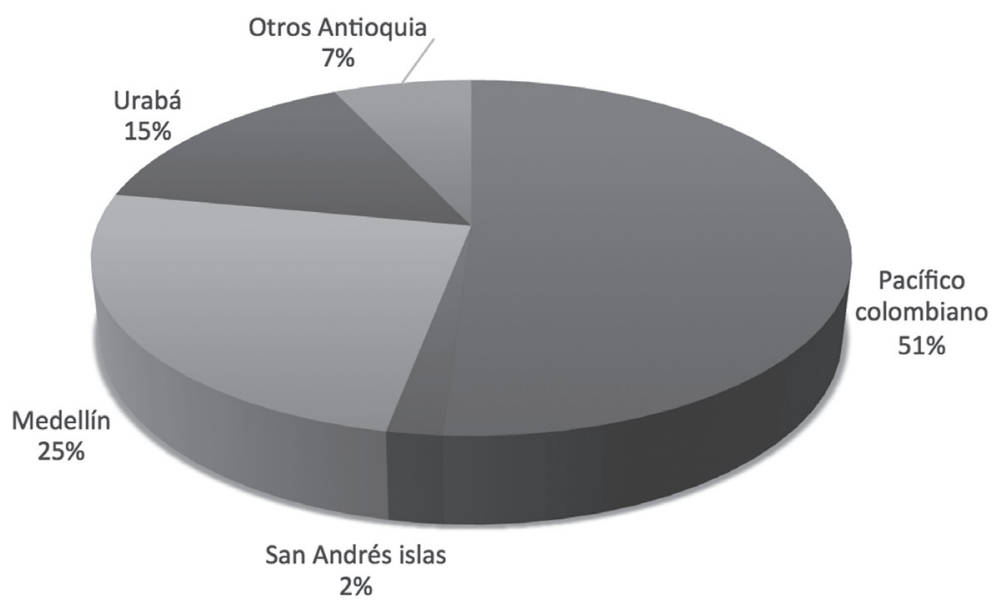

Figura 2. Lugar de origen

El $84 \%$ de los consultados reside en Medellín, donde está ubicada la institución educativa. El $48 \%$ viven con sus padres, y el $24 \%$, con familiares.

A las personas consultadas se les pide que valoren una serie de enunciados relacionados con su identidad, en una escala de aprobación del 1 al 5 (siendo 5 totalmente de acuerdo y 1 totalmente en desacuerdo), como se aprecia en la tabla 1:

Al preguntarles si participan en alguna organización que trabaje el tema afro, solamente el $24 \%$ responde que sí. Por otra parte, entre los consultados se presenta un nivel de abstención similar al de la población colombiana en general, ya que solamente el $44 \%$ participó en las elecciones para elegir representantes al Congreso.

Al ser consultados en cuanto a su acuerdo con que existan medidas a favor de la comunidad afrocolombiana, el $84 \%$ afirma que sí está de acuerdo, a pesar de la elevada abstención. EI total de los consultados afirma que sí existe el racismo en Colombia, pero quienes reconocen haber sido discriminados son el $60 \%$.

Tabla 1. Auto-identificación de las personas consultadas

\begin{tabular}{l|c|c|c|c|c}
\hline & \multicolumn{5}{|c}{ Escala Likert } \\
\cline { 2 - 6 } & $\mathbf{1}$ & $\mathbf{2}$ & $\mathbf{3}$ & $\mathbf{4}$ & $\mathbf{5}$ \\
\hline Me considero afrodescendiente: & $5,4 \%$ & 0 & $5,4 \%$ & $7,2 \%$ & $82 \%$ \\
\hline Me considero afrocolombiano(a): & $3,6 \%$ & $3,6 \%$ & $3,6 \%$ & $7,2 \%$ & $82 \%$ \\
\hline Soy negro(a): & $9 \%$ & $1,8 \%$ & 0 & $7,3 \%$ & $82 \%$ \\
\hline Soy moreno(a): & $43,6 \%$ & $9,1 \%$ & $9,1 \%$ & $10,9 \%$ & $27,3 \%$ \\
\hline Soy mestizo(a): & $76,4 \%$ & $3,6 \%$ & $5,5 \%$ & $5,5 \%$ & $9,1 \%$ \\
\hline
\end{tabular}

\section{Resultados de la autoevaluación de los participantes}

Al final del ejercicio, 80 estudiantes respondieron las preguntas de autoevaluación:

- Todos reconocen que los profesionales de la comunicación deben formarse en el tema de la interculturalidad.
- Todos consideran pertinente el taller sobre comunicación e interculturalidad que se ha realizado en este curso.

- De ellos, 79 afirman que el taller sí reforzó sus competencias para trabajar este tema.

- El $53 \%$ afirma que la parte del taller que más le gustó fue hacerle la encuesta a un estudiante afrodescendiente. 
En la siguiente sesión, los estudiantes debían exponer sus trabajos, para lo cual proyectan el sitio web en el que diseñaron su informe sobre el tema de la discriminación por racismo. Al momento de exponer sus trabajos, los estudiantes leyeron los principales contenidos de texto, mostraron las fotografías que tomaron a los estudiantes que encuestaron y destacaron las fuentes complementarias disponibles en Internet.

Al final de la exposición, surge un debate en el cual los compañeros destacan la importancia de abordar estas temáticas, y de prepararse para asumir un rol positivo ante estas, como profesionales y como ciudadanos.

Mención especial requiere el caso de estudiantes afro, quienes afirmaron sentirse bien trabajando este tema. A pesar de ser una minoría en los grupos de esta asignatura, estos estudiantes participaron en los debates de manera constructiva y afirmaron que sentían que era muy necesario tanto para ellos como para el grupo en general aprender a abordar la diversidad. Algunos, incluso, reconocieron que no conocían las medidas de discriminación positiva que se destacaron en el taller.

\section{Conclusiones}

Se presenta el análisis de la experiencia de diseño y ejecución de un programa formativo en modalidad $b$-learning (aprendizaje bimodal), como una unidad didáctica, dirigida a un grupo de estudiantes de la asignatura "Periodismo Virtual" del pregrado (grado) de Comunicación Social, en Medellín (Colombia).

Por una parte, el propósito del programa parte del reconocimiento de la necesidad de conocer las características, intereses, necesidades y prioridades de una comunidad universitaria conformada por más de 10 mil estudiantes, de los cuales no existe ninguna información sistematizada referente a su identidad étnica.

Y, por otra parte, se trata de dotar de sentido una asignatura que tiene un enfoque técnico, en el cual el estudiante debe apropiarse de las cambiantes y dinámicas herramientas tecnológicas (TIC) para la producción de contenidos periodísticos para la web. Es decir, que cada estudiante pueda trabajar una problemática transversal, pertinente en cuanto a las necesidades del entorno, como la gestión de la diversidad y la superación de la discriminación y el racismo.

En la situación de entrada de los estudiantes, identificada en un primer momento gracias a dos talleres de saberes previos, se destaca que los estudiantes tienen acceso a Internet un número suficiente de horas, tanto para informarse como para su entretenimiento, propio de la etapa juvenil que viven.

En cuanto a su consumo de medios informativos, se identifica la necesidad de reforzar la lectura de prensa y revistas, ya que suelen identificar los informativos de la televisión como principal fuente de información y estos espacios tienden hacia el info-entretenimiento, la espectacularización de la cultura, en detrimento del desarrollo de géneros más elaborados y exigentes y de la profundización y contextualización requeridas por problemáticas sociales complejas y de larga duración, como la discriminación.

Una vez planteada la propuesta para la gestión de la diversidad cultural, se reconoce la necesidad de profundizar en el estudio de la situación de diversidad cultural y étnica de la comunidad universitaria, en futuras investigaciones.

Otro asunto para reflexionar es que la pregunta inicial se enfocara hacia lo afro, que invita a revisar que, de algún modo, la manifestación de diversidad más visible todavía es el color de la piel, a pesar de los cientos de matices que se encuentran en la población latinoamericana, una rica mezcla de mezclas. Y, sin embargo, en este tema queda mucho por luchar, por lo que se reconoce que se trata de procesos educativos de larga duración. Ejemplo de ello es ver cómo, a pesar de la existencia de una ley que garantiza que la población afrodescendiente debe contar con dos congresistas, en las últimas elecciones (2014) se eligieron como representantes afro dos personas que ni pertenecen a este grupo poblacional ni han trabajado en organizaciones que reivindiquen los derechos afro, según denuncia la prensa. 
En las elecciones mencionadas tampoco se logró paridad de géneros, a pesar de la Ley de Cuotas que obliga a los partidos a que mínimo el $30 \%$ de sus listas estén conformadas por mujeres.

Como docente, la experiencia que deja la aplicación de este programa es muy positiva, ya que el grupo de estudiantes manifiesta haber quedado motivado para profundizar en este tipo de temáticas, propias de la diversidad humana, y para seguir mejorando su proceso de apropiación de las TIC para generar contenidos que sean usables y eficaces. Es pertinente que este curso se haya planteado como un taller bimodal, ya que los estudiantes necesitaron mucho acompañamiento durante el proceso de selección de las fuentes de información y la elaboración de su publicación electrónica sobre la discriminación en un sitio web de la plataforma Webnode $\AA$.

Se destaca que a los estudiantes lo que más les llamó la atención del trabajo de aplicación fue la oportunidad de abordar a un estudiante afrodescendiente de la misma institución, a través de un cuestionario previamente elaborado por el docente. Pero el cuestionario fue solamente un instrumento guía, de acuerdo con la sustentación de los estudiantes, para propiciar una conversación más abierta con la persona encuestada, de manera que se complementó con un ejercicio de entrevista, mucho más rico, porque permitió acceder a explicaciones más profundas por parte de los estudiantes afrodescendientes abordados, con quienes normalmente no hablarían.

La pertinencia de la estrategia de aprendizaje bimodal también se ve justificada, debido a que una parte significativa del grupo de estudiantes exige un proceso de acompañamiento muy personalizado y algo paternalista, en el cual van verificando con su profesor, a cada paso, que no estén perdidos, aunque tengan a su disposición toda la información, las guías, rúbricas y bibliografía, en diferentes escenarios virtuales (como la plataforma Moodle o el sitio web del docente). Se confirma la necesidad de fortalecer su autonomía y criterio propio para que puedan aprovechar mejor las herramientas de educación virtual y a distancia, y es necesario seguir indagando en cuanto a experiencias y metodologías que propicien este proceso.

\section{Referencias bibliográficas}

- Alcaldía de Medellín (2010). "Desplazamiento forzado por la violencia en la ciudad de Medellín". Recuperado de http://www.medellin. gov.col

- Araya, Carlos (2007) Diseño, ejecución y evaluación de un curso bimodal en la educación superior. Actualidades Investigativas en Educación Volumen 7, Número Especial (noviembre 2007), 1-22. Recuperado de http:// dx.doi.org/10.15517/aie.v7i4.9303

- Ariño, A. (1997). Sociología de la cultura. Barcelona: Ariel. 235.

- Cabero, Julio y Llorente, María (2008) Del eLearning al Blended Learning: nuevas acciones educativas. Quaderns digitals N. ${ }^{\circ} 51$. Recuperado de http://www.quadernsdigitals.net/ index. php?accionMenu=hemeroteca. VisualizaNumeroRevistalU.visualiza\&numeroRevista_id $=773 \&$ PHPSESSID=cb42b5b9194a3b4470427 f2a40140e2a

- Carabalí, A. (2007). La etnoeducación campo de articulaciones necesarias. Boletín Antropológico, 25, (69), 57-82. Universidad de los Andes, Venezuela. Recuperado de http:// www.redalyc. org/articulo.oa?id=71206903

- DANE (2007) Colombia una nación multicultural: Su diversidad étnica. Recuperado de https:// www.dane.gov.co/files/censo2005/etnia/sys/ colombia_nacion.pdf

- ECLAC. (2013) Observatorio para la Sociedad de la información en Latinoamérica y el Caribe. Recuperado de http://websie.eclac.cl/scripts/ broker.exe

- García-canclini, N. (1982). Las culturas populares en el capitalismo. La Habana: Casa de las Américas.

- García-canclini, N. (2004). "Diferentes, desiguales o desconectados." En: Revista CIDOB d'Afers Internacionals, 66-67, 2004. Zaragoza: CIDOB. 113-133.

- Guzmán, C. y otros. (2009). Deserción estudiantil en la educación superior colombiana. Bogotá, Ministerio de Educación Nacional.

- Hernández, G. (2005) La condición global. 1 y 2. Valencia: Germania.

- Lotero, G. (2014a) Enseñanza del periodismo virtual: desafíos y criterios para la producción de contenidos para la web. En: Lotero, G. (Comp.) Investigación en comunicación-educación y publicidad. Medellín, Funlam. Recuperado de http://www.funlam.edu.co/uploads/ 
fondoeditorial/seminario_comunicacion_educacion_2013-2014.pdf

- Lotero, G. (2014b). Educación para la ciudadanía en la era de las wikis. Poliantea, 10(19), 199212. DOI: http://dx.doi.org/10.15765/plnt. v10i19.579

- Ministerio de Educación de Colombia. (2004). Estándares Básicos de Competencias Ciudadanas. Bogotá.

- ONU. (1966a) Pacto Internacional de Derechos civiles y políticos. Washington: ONU. Recuperado de http://www2.ohchr.org/spanish/ law/ccpr.htm

- $\quad$ ONU. (1966b) Pacto Internacional de Derechos económicos, sociales y culturales. Washington: ONU. Recuperado de http://www2.ohchr.org/ spanish/law/cescr.htm
- Pereira, Gustavo. (2009) Preferencias adaptativas como bloqueo de la autonomía. En: CORTINA, A. y Pereira, G. (2009). Pobreza y libertad: Erradicar la pobreza desde el enfoque de Amartya Sen. Madrid: Tecnos.

- PNUD. (2010) Informe Regional sobre Desarrollo Humano para América Latina y el Caribe. Santiago: PNUD.

- Red de Ciudades Cómo Vamos (2014) Informe de Calidad de Vida 2011-2013. Recuperado de http://redcomovamos.org/descarga-el-informede-calidad-de-vida-2011-2013-comparado-en14-ciudades-colombianas/

- SEN, A. (2007). Primero la gente. Barcelona: Deusto.

- SEN, A. (2000) Desarrollo y libertad. Barcelona: Planeta. 440. 